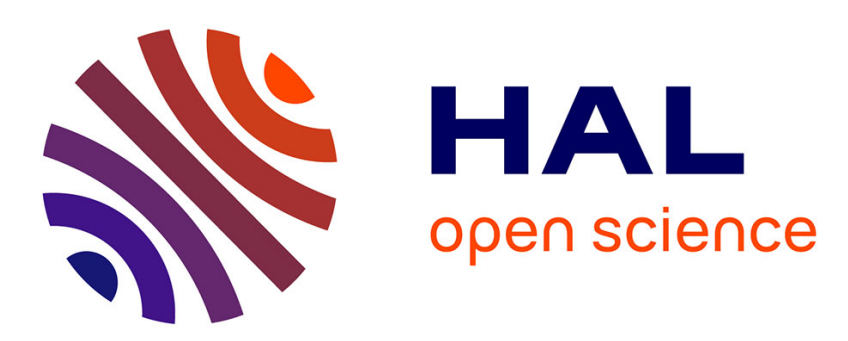

\title{
Experimental analysis of non linear tolerance dependency of multicarrier modulations versus bandwidth efficiency
}

\author{
A Carbo, J Renaudier, R Rios-Müller, P Tran, G Charlet
}

\section{To cite this version:}

A Carbo, J Renaudier, R Rios-Müller, P Tran, G Charlet. Experimental analysis of non linear tolerance dependency of multicarrier modulations versus bandwidth efficiency. 31st European Conference on Optical Communication, Sep 2005, Valencia, Spain. pp.1 - 3, 10.1109/ECOC.2015.7341901 . hal01525707

\section{HAL Id: hal-01525707 \\ https://hal.science/hal-01525707}

Submitted on 22 May 2017

HAL is a multi-disciplinary open access archive for the deposit and dissemination of scientific research documents, whether they are published or not. The documents may come from teaching and research institutions in France or abroad, or from public or private research centers.
L'archive ouverte pluridisciplinaire HAL, est destinée au dépôt et à la diffusion de documents scientifiques de niveau recherche, publiés ou non, émanant des établissements d'enseignement et de recherche français ou étrangers, des laboratoires publics ou privés. 


\title{
Experimental Analysis of Non Linear Tolerance Dependency of Multicarrier Modulations versus Bandwidth Efficiency
}

\author{
A. Carbo, J. Renaudier, R. Rios-Müller, P. Tran, G. Charlet
}

Bell Labs Alcatel-Lucent, 91620 Nozay, France, Alexis.Carbo Meseguer@Alcatel-Lucent.com

\begin{abstract}
We report on the experimental comparison of the non linear tolerance of multicarrier and single-carrier modulations in WDM scenarios while varying the bandwidth efficiency. Non linear tolerance improvement of multicarrier approach decreases with bandwidth efficiency.
\end{abstract}

\section{Introduction}

Although linear effects in coherent receivers can be compensated using digital signal processing (DSP), the mitigation of nonlinear impairments remains a major issue in optical fiber transmission systems ${ }^{1}$. Some techniques have been proposed in order to combat these impairments, such as digital back propagation ${ }^{2,3}$, perturbative non linear compensation ${ }^{4,5}$, but requiring high computational complexity. Another technique, based on dividing high baud rate signals into several lower baud rate subcarriers, has been investigated to increase the tolerance to nonlinearities in uncompensated optical transmission systems. Such a multicarrier modulation scheme was reported to provide reach improvements up to $22 \%$ in quadrature phase shift keying (QPSK) modulations at 24 GBaud $^{6}$. However, this experimental result was obtained in a singlechannel transmission without accounting for cross non linearities stemming from adjacent WDM channels in optical systems. In the meantime numerical and analytical studies have reported $^{7,8}$ on the impact of the symbol rate onto non linear tolerance in WDM systems, whose results tend to indicate that symbol rates below $10 \mathrm{GBd}$ may provide optimum performance thanks to improved non linear tolerance.

In this paper, we report on the experimental assessment of non linear tolerance of multicarrier and single-carrier modulations on an uncompensated transmission link made of 100$\mathrm{km}$ long spans of standard single-mode fiber (SSMF). Using polarization division multiplexed (PDM) quadrature phase shift keying (QPSK) data the performance of multicarrier and singlecarrier modulations are compared in a WDM transmission system composed of sixty-four 50$\mathrm{GHz}$ spaced channels. The results are obtained with two symbol rates, namely 24 and $48 \mathrm{GBd}$, in order to vary the bandwidth efficiency of the systems.

\section{Experimental setup}

We conducted an experimental comparison of the non linear tolerance of single-carrier, 4carrier and 8-carrier modulations in $50-\mathrm{GHz}$ spaced WDM systems while varying the bandwidth efficiency, which is defined as $\eta=R / \Delta f$ with $\mathrm{R}$ being the symbol rate of one WDM channel and $\Delta f$ being the spacing between channels. In the case of a multicarrier modulation scheme, $\mathrm{R}$ corresponds to the total baud rate of all the subcarriers. As schematically described in Fig. 1, for multicarrier modulation we consider that the spacing between carriers is equal to their bandwidth, namely $R \cdot(1+\alpha) / N$, with $\mathrm{N}$ being the number of carriers and $\alpha$ a generic roll-off factor. Therefore, the total bandwidth of multicarrier modulated channels is the same as the bandwidth of the single-carrier modulated ones, and they experienced the same WDM bandwidth efficiency. Considering a constant channel spacing of $50 \mathrm{GHz}$, we used two total symbol rates of $24 \mathrm{GBd}$ and $48 \mathrm{GBd}$ so that the bandwidth efficiency varies from $\eta=0.48$ to $\eta=0.96$ respectively.
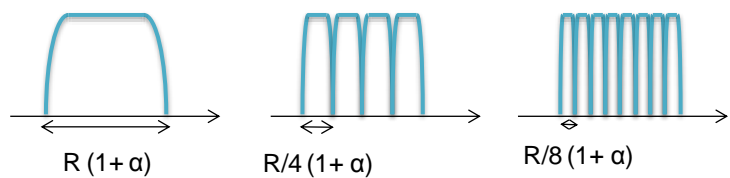

Fig. 1: Single-carrier and multicarrier signals for a roll-off factor $\alpha$ and a symbol-rate $R$.

Our test-bed, depicted in Fig. 2-a), consists of
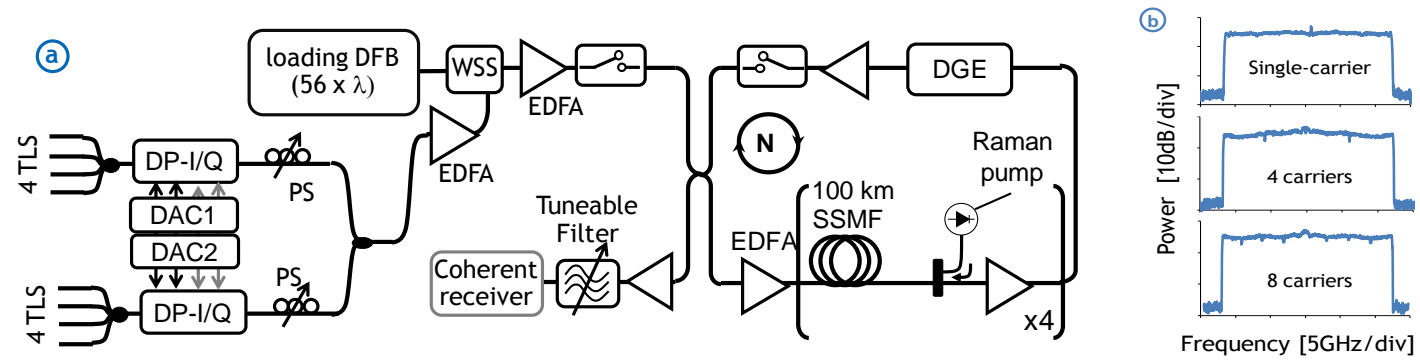

Fig. 2: (a) WDM transmission setup and (b) Spectra of the 24 GBd Signals for Singlecarrier and Multicarrier (4 and 8 carriers). 
eight tunable laser sources (TLS) with $100 \mathrm{kHz}$ linewidth, including the laser under test at $1545.72 \mathrm{~nm}$, plus fifty six loading DFB channels. All sixty four channels are spaced by $50 \mathrm{GHz}$ as aforementioned. Our eight TLS are separated into odd and even sets and sent to separate PDM I/Q modulator driven by distinct digital-toanalog converters (DAC) operating at 88GSamples/s. These two sets are then passed into separate low-speed $(<10 \mathrm{~Hz})$ polarization scramblers (PS) and spectrally combined through an optical coupler. Loading DFB channels are modulated independently to generate the same format, modulation scheme and symbol rate as our eight TLS channels.

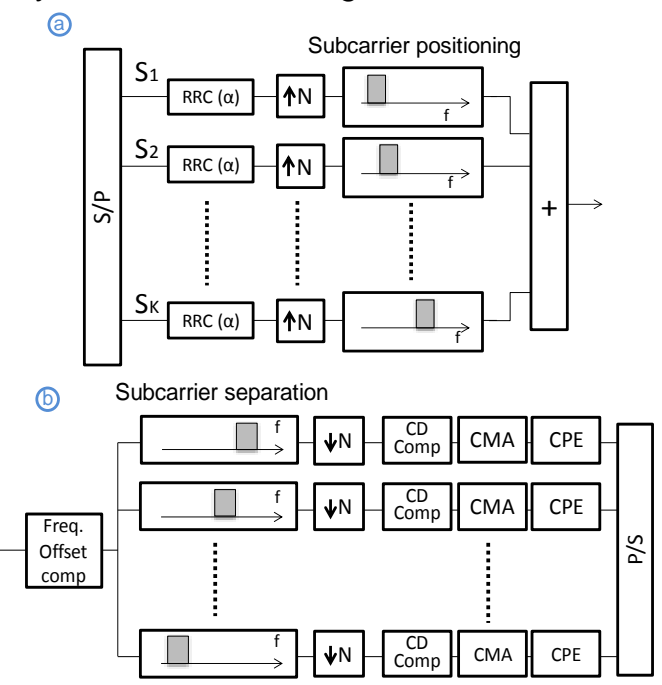

Fig. 3: Multicarrier transmitter and receiver DSP

For single-carrier modulation, QPSK symbol sequences are generated using multiple delayed decorrelated sequences of $2^{15}$ bits and filtered by a root-raised cosine (RRC) filter with a roll-off factor of 0.01 . In the case of multicarrier generation, the subcarriers are generated digitally according to Fig. 3-a). Symbol sequences are generated using multiple delayed decorrelated sequences of $2^{15}$ bits which are divided into $\mathrm{N}$ streams, where $\mathrm{N}$ is the number of subcarriers desired. We have computed the correlation of these sequences to ensure that the correlation peak between adjacent carriers is $30 \mathrm{~dB}$ below the power of the signal. The sequences of each subcarrier are filtered by the RRC filter with a roll-off factor of 0.01 , up converted in frequency domain to their corresponding intermediate frequencies before summation and time domain conversion of the overall multicarrier spectrum. Fig. 2-b) shows the generated spectrum of single, 4- and 8carrier signals at the symbol of $24 \mathrm{GBd}$.

After being generated, all channels are recombined using a wavelength selective switch (WSS). The resulting multiplex is boosted through a single stage erbium doped fiber amplifier (EDFA) and sent into the recirculating loop. The loop consists of four $100 \mathrm{~km}$-long spans of SSMF. No dispersion-compensating fiber is used here. Hybrid Raman-EDFA optical repeaters compensates for fiber losses. The Raman pre-amplifier is designed to provide $\sim 10 \mathrm{~dB}$ on-off gain. Dynamic gain equalization (DGE) is performed thanks to a $50-\mathrm{GHz}$ grid WSS inserted at the end of the loop.

At the receiver side, the channel under test at $1545.72 \mathrm{~nm}$ is selected by a tunable filter and sent into a polarization-diversity coherent mixer feeding four balanced photodiodes. Their electrical signals are sampled at $80 \mathrm{GS} / \mathrm{s}$ by a real-time digital oscilloscope having a $33-\mathrm{GHz}$ electrical bandwidth. For each measurement, five different sets of $40 \mu$ s are stored. Thanks to polarization scrambling, each recording corresponds to an arbitrary state of polarization at the input of the link. The received samples are processed off-line in a computer to recover the transmitted data as described in Fig. 3-b). Frequency offset compensation is performed in the frequency domain before subcarriers separation. Then digital signal processing of each subcarrier includes chromatic dispersion compensation, polarization demultiplexing and carrier phase recovery (CPE). The length of the CPE algorithm has been optimized for each measurement. Finally their bit error rates (BER) are computed and averaged out to obtain the BER of the channel under test, subsequently converted into $Q^{2}$ factor.

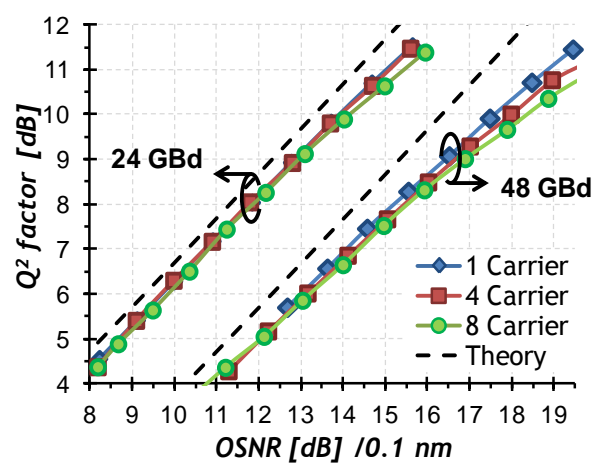

Fig. 4: Back-to-back characterization at 24 and $48 \mathrm{GBd}$.

\section{Experimental results}

Fig. 4 shows the performance in back-to-back of single-carrier, 4-subcarrier and 8-subcarrier configurations with 24 and 48 GBd PDM-QPSK data together with theoretical expectations. At both symbol rates, although the 8-carrier scheme exhibits higher OSNR penalties at $\mathrm{Q}^{2}$ factor above $10 \mathrm{~dB}$, which can be attributed to its highest peak-to-average power ratio, it does not exhibit OSNR penalty with respect to single 
and 4-carrier schemes for lower $\mathrm{Q}^{2}$ factors.

Next we investigated the tolerance to non linear impairments of single-carrier and multicarrier systems depending on the bandwidth efficiency using our recirculating loop. First we measured their performance using PDM-QPSK modulation and a symbol rate of 24 GBd, leading to a bandwidth efficiency of 0.48 . Fig.5 shows the evolution of the $Q^{2}$ factor as a function of channel launch power after 20 loop round-trips, corresponding to $8000 \mathrm{~km}$. It can be observed that the 4-carrier and the 8-carrier signals exhibit an optimum channel power near to $-1.5 \mathrm{dBm}$, which is $1 \mathrm{~dB}$ higher than the single-carrier one. Moreover, the performance in this optimum power is $7.4 \mathrm{~dB}$ for Single-carrier, $7.8 \mathrm{~dB}$ for 4-Carriers and $7.6 \mathrm{~dB}$ for 8-Carriers, these results indicate that the multicarrier signals outperforms the single-carrier in $0.4 \mathrm{~dB}$ and $0.2 \mathrm{~dB}$ respectively for 4-Carriers and 8Carriers. It also shows that the best results are obtained using 4-Carriers that corresponds with a baud rate equal to $6 \mathrm{GBd}$ per carrier. The 0.4 $\mathrm{dB}$ performance improvement would be translated into $8 \%$ increase in reach with respect to SC.

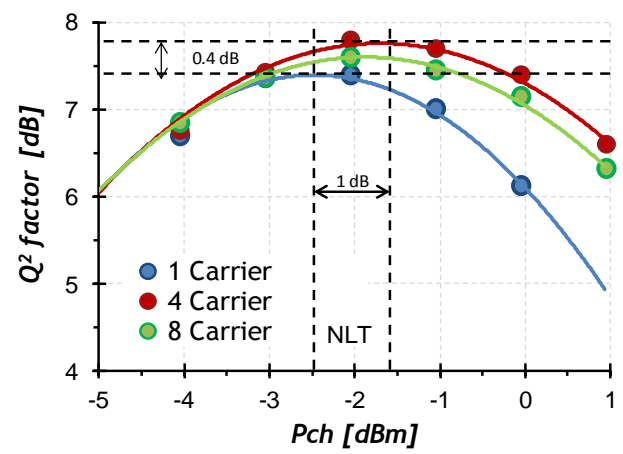

Fig. 5: $Q^{2}$ Factor versus launch power after $8000 \mathrm{~km}$ for single-carrier, 4-carrier and 8-carrier signals with $\boldsymbol{\eta}=\mathbf{0 . 4 8}$.

Then, we modulated all $50-\mathrm{GHz}$ spaced channels with the symbol rate of $48 \mathrm{GBd}$, thus doubling the bandwidth efficiency of the system at 0.96 . Fig. 6 shows the performance of the single-carrier, 4-carrier and 8-carrier signals versus launch channel power at the transmission distance of $6400 \mathrm{~km}$, which corresponds to 16 loops. We observe in this figure that all the signals exhibit similar tolerance to non linear impairments and the same optimum channel power of $-1 \mathrm{dBm}$. Contrary to the previous experiment, the three configurations show nearly identical $\mathrm{Q}^{2}$ factor around $7.5 \mathrm{~dB}$ in the optimum power. This result indicates that performance appears weakly dependent on symbol rate when bandwidth efficiency increases close to one in WDM systems.

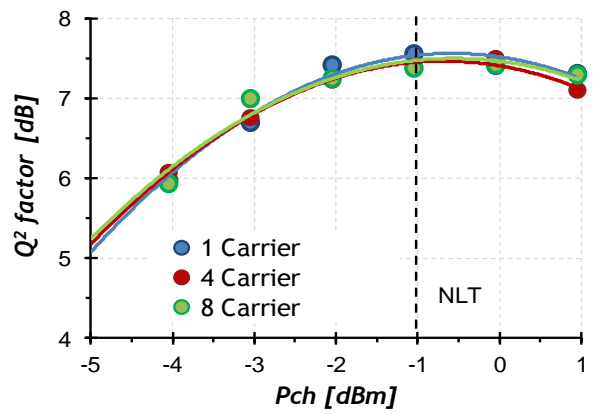

Fig. 6: $Q^{2}$ Factor versus launch power after $6400 \mathrm{~km}$ for single-carrier, 4-carrier and 8-carrier signals with $\boldsymbol{\eta}=\mathbf{0 . 9 6}$.

\section{Conclusions}

We have assessed experimentally the benefits of using the multicarrier approach for the mitigation of non linearities in WDM transmission systems. As compared to singlecarrier modulation, the multicarrier scheme with 4 carriers enables an $8 \%$ increase in transmission reach over an uncompensated transmission link with a bandwidth efficiency of 0.48 . We also showed that this benefit is compromised when bandwidth efficiency is increased up to 0.96 . Therefore, these results indicate the dependency of non linear tolerance versus symbol-rate tends to disappear as bandwidth efficiency increases. These results are not in perfect agreement with recently reported results by Poggiolini et $\mathrm{al}^{8}$ and further investigations might be required.

\section{References}

[1] A. Mecozzi et al, "Nonlinear Shannon Limit in Pseudolinear Coherent Systems", JLT, Vol. 30, no. 12, p. 2011-2024 (2012).

[2] E. Ip and J.M. Kahn, "Compensation of Dispersion and Nonlinear Impairments Using Digital Backpropagation" JLT, Vol. 26, no. 20, p.3416-3425 (2008).

[3] Y. Gao, et al, "Assessment of Intrachannel Nonlinear Compensation for $112 \mathrm{~Gb} / \mathrm{s}$ Dual-Polarization 16QAM Systems", JLT Vol. 30, no. 24, p. 3902-3910 (2012).

[4] Z. Tao et al., "Multiplier-Free Intrachannel Nonlinearity Compensating Algorithm Operating at Symbol Rate", JLT, Vol. 29, no. 17, p. 2570-2576 (2011).

[5] Y. Gao et al. "Reducing the Complexity of Nonlinearity Pre-compensation Using Symmetric EDC and Pulse Shaping", Proc. ECOC, PD 3.E.5, London (2013)

[6] M. Qiu, Q. Zhuge and al, "Digital subcarrier multiplexing for fiber nonlinearity mitigation in coherent optical communication systems", Optics Express, Vol.22, no.15, p. 18770-18777 (2014).

[7] A. Bononi and al, "Performance Dependence on Channel Baud-Rate of Coherent Single-carrier WDM Systems", Proc. ECOC, Th.1.D.5, London (2013).

[8] P. Poggiolini et al, "Analytical results on system maximum reach increase through symbol rate optimization", Proc. OFC15, Th.3.D.6, Los Angeles (2015). 